\title{
RELACIONES POSITIVAS CON OTRAS PERSONAS Y AUTOEFICACIA EN ESTUDIANTES UNIVERSITARIOS
}

\author{
María del Mar Ferradás Canedo \\ Departamento de Psicología \\ Universidade da Coruña (España) \\ mar.ferradasc@udc.es \\ Carlos Freire Rodríguez \\ Departamento de Psicología \\ Universidade da Coruña (España)
}

Recepción Artículo: 24 enero 2020

Admisión Evaluación: 4 marzo 2020

Informe Evaluador 1: 1 enero 2020

Informe Evaluador 2: 1 enero 2020

Aprobación Publicación: 20 abril 2020

\section{RESUMEN}

La autoeficacia constituye una de las variables motivacionales más determinantes de las conductas de logro de los estudiantes, influyendo significativamente sobre el nivel de esfuerzo y persistencia en las tareas, así como en el rendimiento académico. Su importante contribución en el ámbito académico ha promovido un creciente interés por la identificación de los recursos psicológicos que favorecerían las expectativas de autoeficacia en el contexto académico. En consonancia con este planteamiento proactivo, el objetivo del presente estudio fue analizar la relación entre la autoeficacia y las relaciones positivas con otras personas, esta última erigida en una de las dimensiones esenciales del bienestar psicológico humano. En la investigación participaron 630 estudiantes (427 mujeres y 203 hombres) de la Universidade da Coruña (España), con edades comprendidas entre los 18 y los 46 años $(M=21.17 ; D T=3.31)$. Se llevó a cabo un estudio no experimental con un diseño de cohortes transversales. Se establecieron tres niveles (bajo, medio y alto) en la variable relaciones positivas con otros, tomando como puntos de corte los percentiles 33 y 66 . Las diferencias en autoeficacia entre estos tres niveles se determinaron mediante un ANOVA de un factor. Los resultados evidenciaron diferencias estadísticamente significativas entre los tres niveles, de manera que el grupo con niveles más elevados de relaciones positivas con otros mostró niveles de autoeficacia general más altos que los grupos medio y bajo. La magnitud del efecto fue media. Estos hallazgos sugieren que disponer de lazos afectivos significativos con otras personas favorecería en el estudiantado universitario creencias de competencia autopercibida más elevadas para hacer frente a su día a día.

Palabras clave: relaciones positivas con otras personas; autoeficacia; bienestar psicológico; estudiantes universitarios; psicología positiva 


\section{ABSTRACT}

Positive relationships with others and self-efficacy in university students. Self-efficacy constitutes one of the most decisive motivational variables of students' achievement behaviors, significantly influencing the level of effort and persistence in tasks, as well as academic performance. Its important contribution in the academic field has promoted a growing interest in the identification of psychological resources that would favor selfefficacy expectations in the academic field. In line with this proactive approach, the objective of the present study was to analyze the relationship between self-efficacy and positive relationships with others, the latter erected in one of the core dimensions of psychological well-being. The research involved 630 students (427 women and 203 men) from the University of Corunna (Spain), aged between 18 and 46 years $(M=21.17 ; D T=3.31)$. A nonexperimental study was conducted with a transverse cohort design. Three levels (low, medium, and high) were established in the variable positive relationships with others, taking the 33 and 66 percentiles as cut-off points. The differences in self-efficacy between these three levels were determined by a one-way ANOVA. The results showed statistically significant differences between the three levels, so that the group with higher levels of positive relationships with others showed higher levels of general self-efficacy than the middle and low groups. The magnitude of the effect was medium. These findings suggest that having significant emotional ties with other people would favor higher students' self-perceived competencies to face their day-to-day life.

Keywords: positive relationships with others; self-efficacy; psychological well-being; university students; positive psychology

\section{ANTECEDENTES}

Las expectativas de autoeficacia constituyen el elemento central dentro de la Teoría Cognitiva Social desarrollada por Bandura $(1977,1997)$. En palabras del propio autor, la autoeficacia podría definirse como: "las creencias de las personas en sus capacidades para movilizar la motivación, los recursos cognitivos y el curso de acción necesarios para ejercer control sobre los acontecimientos de sus vidas" (Wood y Bandura, 1989, p. 364). En consecuencia, la autoeficacia constituye una creencia autorreferida decisiva para la consecución de metas y tareas personales, dado que, si las personas creen que no tienen poder para producir resultados, no harán ningún intento para lograr que esto suceda.

Atendiendo a estas consideraciones, en lo tocante al plano académico, la autoeficacia adquiere un papel especialmente relevante en la conducta motivada del estudiante, influyendo notablemente en sus procesos cognitivos, cursos de acción y reacciones emocionales. En este sentido, un prolífico cuerpo de trabajos ha evidenciado el papel decisivo que juega esta expectativa autorreferida en el compromiso con las tareas y la consecución de las metas deseadas, así como en los niveles de esfuerzo y persistencia ante las dificultades, en la autorregulación y en el rendimiento académico (Alhadabi y Karpinski, 2020; Honicke y Broadbent, 2016; Ritchie, 2016; Schunk y Pajares, 2010; Shih, 2019; Zumbrunn, Broda, Varier y Conklin, 2019).

Habida cuenta de la importante contribución de la autoeficacia en el contexto académico, desde la Psicología positiva existe un creciente interés por identificar aquellos recursos psicológicos que favorecerían el desarrollo de las creencias de competencia en el estudiantado. En línea con otros trabajos que han adoptado este enfoque proactivo (e.g., Freire y Ferradás, 2019), el presente estudio pretende analizar la relación entre las relaciones positivas con otras personas y la autoeficacia en el contexto universitario.

El ampliamente aceptado modelo multidimensional del bienestar psicológico de Ryff (Ryff, 1989, 2013; Ryff y Keyes, 1995) plantea que las relaciones positivas con otras personas son un pilar esencial para la consecución del bienestar psicológico. En este modelo, las relaciones positivas con otras personas se definen como la condición en la que el individuo posee relaciones íntimas, satisfactorias y de plena confianza con otros individuos. Este tipo de vínculos interpersonales "están universalmente considerados como centrales para una existencia óptima" (Ryff y Singer, 2000, p. 30). En efecto, esta querencia por la afiliación social es universal, en tanto en cuanto parece tener una base neurobiológica (Rowe, 1996) y, por tanto, sería tan básica para el bienestar psicológico de las personas como lo son el alimento y el agua para nuestro bienestar físico (Baumeister y Leary, 1995). 
En el contexto universitario, diversos trabajos han revelado la existencia de una relación positiva y directa entre las interacciones positivas con otros estudiantes y el desarrollo psicosocial del individuo, en términos de madurez, autonomía, sentimiento de pertenencia, integración e identificación grupal (Adams, Ryan y Keating, 2000; Zander, Brouwer, Jansen, Crayen y Hannover, 2018). Tal es así que la adaptación social de los estudiantes universitarios se revela como un factor tan importante como los meramente académicos a la hora de predecir el grado de persistencia (c.f., Mallinckrodt, 1988). Y al contrario, entre las principales causas que explican los problemas aducidos por los estudiantes durante el primer año de carrera se encuentran las dificultades de adaptación social, problemática que se traduce en sentimientos de añoranza y soledad (Fiori y Consedine, 2013; Lau, Chan y Lam, 2018). A su vez, el soporte familiar parece erigirse en un elemento importante y complementario al apoyo brindado por amigos y otros estudiantes (Feldman et al., 2008). La familia proporcionaría soporte a nivel efectivo (apoyo económico, facilitación de recursos, etc.) y afectivo, fundamentalmente en períodos de transición como el inicio en la etapa universitaria o la finalización de la misma, contribuyendo a afrontar el estrés y favoreciendo una mayor satisfacción vital (DeRosier, Frank, Schwartz y Leary, 2013; Schnettler et al., 2017). En esta línea, el estudio realizado por Wright, Perrone-McGovern, Boo y White (2014) con universitarios norteamericanos concluye que la percepción de relaciones sociales de apoyo favorece la autoeficacia del estudiante a la hora de tomar decisiones a lo largo de su trayectoria académica. Tomando como referencia los resultados de este trabajo, el objetivo del presente estudio es analizar si diferentes niveles de relaciones positivas con otras personas (alto, medio y bajo) se relacionan diferencialmente con la autoeficacia general de los estudiantes universitarios. Se hipotetiza que aquellos estudiantes con niveles más elevados de vínculos positivos con otros evidenciarán creencias de autoeficacia general significativamente mayores que los estudiantes con niveles medios y bajos de relaciones positivas.

\section{MÉTODO}

\section{Participantes}

El estudio se efectuó con una muestra de 630 estudiantes $\left(M_{\text {edad }}=21.17 ; D T=3.31\right)$ de la Universidade da Coruña (España), seleccionados mediante un muestreo por conveniencia. Del total de titulaciones ofertadas por esta Universidad, 15 aceptaron voluntariamente participar en el estudio: Educación Infantil (11.9\%), Educación Primaria (4.3\%), Educación Física (4.6\%), Educación Social (7.6\%), Audición y Lenguaje (3.2\%), Logopedia (4.8\%), Psicopedagogía (0.8\%), Fisioterapia (6.8\%), Enfermería (6.3\%), Podología (7.1\%), Derecho 11.4\%), Sociología (7.3\%), Ciencias de la Actividad Física y el Deporte (7.3\%), Arquitectura Superior (11\%), Arquitectura Técnica (6.8\%) e Ingeniería de Caminos, Canales y Puertos (6\%). Atendiendo al género, 427 participantes $(67.8 \%)$ eran mujeres. Respecto al curso, el 30\% eran estudiantes de primer curso; el $5.2 \%$, de segundo; el $10.8 \%$, de tercero; el $28.4 \%$, de cuarto; y el $25.6 \%$ cursaban quinto.

\section{Instrumentos}

Relaciones positivas con otras personas. La variable relaciones positivas con otras personas se evaluó mediante la subescala de relaciones positivas con otros (positive relationships with others) incluida en la adaptación española de las Escalas de Bienestar Psicológico de Ryff (Díaz et al., 2006). La subescala incluye cinco ítems ("Siento que mis amistades me aportan muchas cosas"), sobre los que los participantes debían mostrar su grado de acuerdo seleccionando uno de los cinco puntos contenidos en una escala Likert ( $1=$ En total desacuerdo $-5=$ Totalmente de acuerdo). La consistencia interna del instrumento evidenciada en nuestro estudio fue de $\alpha=.77$, en consonancia con otros estudios efectuados con estudiantes universitarios (e.g., Freire, Ferradás, Núñez y Valle, 2017).

Autoeficacia. Para la medida de la autoeficacia se utilizó la Escala de Autoeficacia General (Baessler y Schwarcer, 1996). Se trata de un instrumento que consta de diez ítems (e.g., "Tengo confianza en que podría manejar eficazmente acontecimientos inesperados"), a los que los participantes debían responder entre cinco 
alternativas de respuesta dentro de una escala Likert ( $1=$ "Nunca" $-5=$ "Siempre"). En el presente trabajo, esta escala evidenció una consistencia interna adecuada $(\alpha=.91)$, en línea con trabajos precedentes con estudiantes universitarios (e.g., Sanjuán, Pérez y Bermúdez, 2000, en el que $\alpha=.87$ ).

\section{Procedimiento}

La investigación se llevó a cabo en consonancia con los criterios éticos que rigen las investigaciones con seres humanos, con consentimiento informado por escrito de todos los participantes, tal y como se expone en la Declaración de Helsinki (World Medical Association, WMA, 2013). Previamente a la recogida de datos, se informó a los participantes acerca de los objetivos y características del estudio y se les solicitó su colaboración desinteresada. Asimismo, se les garantizó el anonimato y la confidencialidad de sus respuestas, indicándoseles que su decisión de participar o no hacerlo en el estudio carecería de repercusión alguna. Los datos obtenidos fueron recogidos en las aulas donde los participantes reciben su formación académica, dentro del horario de clases y en una única sesión sin límite de tiempo. Aquellas personas que voluntariamente declinaron participar en el estudio abandonaron el aula antes del inicio de la prueba.

\section{Análisis de datos}

En primer término, se analizaron los estadísticos descriptivos (media, desviación típica, asimetría y curtosis) de las variables del estudio, así como la matriz de correlaciones (Pearson). A continuación, se determinaron, mediante un ANOVA de un factor, las diferencias entre los tres niveles (alto, medio y bajo) de relaciones positivas con otros respecto a la autoeficacia, siendo considerada esta última la variable dependiente. Previamente se establecieron los tres grupos en en los que fue categorizada la variable relaciones positivas con otras personas. Para ello, se tomaron como puntos de referencia las puntuaciones terciles (<P33, grupo bajo; entre P33 y P66, grupo medio; > P66, grupo alto). Las comparaciones a posteriori se analizaron mediante las pruebas de Scheffé y de Games-Howell, optándose por una o por otra en función de si en cada caso era pertinente asumir o no homogeneidad en las varianzas Se estimó también la magnitud del efecto mediante el estadístico $d$ de Cohen. Siguiendo las recomendaciones de Cohen (1988), se considera como pequeño aquel efecto $d \leq 0.20$; medio, entre $d>0.20$ ) y $d<0.80)$; y grande, si $d \geq 0.80$.

\section{RESULTADOS}

\section{Estadísticos descriptivos y correlaciones}

En la Tabla 1 se muestran los estadísticos descriptivos y los índices de correlación entre las variables relaciones positivas con otros y autoeficacia general. De acuerdo con los resultados recogidos en ella, se observan niveles promedio altos en la variable relaciones positivas con otros $(M=4.24)$ y moderadamente altos en autoeficacia ( $M=3.38$, en autoeficacia). Por otra parte, los valores de los indicadores de dispersión (asimetría y curtosis) son respectivamente inferiores $\mathrm{a} \pm 2 \mathrm{y} \pm 7$ en las dos variables, lo que, de acuerdo con los criterios de Finney y DiStefano (2006), indica que los datos se ajustan a una distribución normal. Respecto al análisis de correlaciones (Pearson), se observa una correlación positiva significativa entre relaciones positivas con otros y autoeficacia general $(r=.23, p<.01)$. 
Tabla 1

Medias, desviaciones típicas, asimetría, curtosis y matriz de correlaciones ( $N=630)$

\begin{tabular}{|c|c|c|}
\hline & 1 & 2 \\
\hline $\begin{array}{l}\text { 1. RE_PO } \\
2 . \\
\text { AUTOE }\end{array}$ & $.23 * *$ & - \\
\hline$M$ & 4.24 & 3.38 \\
\hline$D T$ & 0.67 & 0.68 \\
\hline Asimetría & -1.06 & 0.01 \\
\hline Curtosis & 1.17 & -0.37 \\
\hline
\end{tabular}

Nota. RE_PO = Relaciones positivas con otros; AUTOE = Autoeficacia.

Escala de medida de relaciones positivas con otros ( $1=$ En total desacuerdo, $2=$ Algo en desacuerdo, $3=\mathrm{Ni}$ en desacuerdo ni de acuerdo, 4 = Algo de acuerdo, 5 = Totalmente de acuerdo); Escala de medida de autoeficacia $(1=$ Nunca, 2 = Pocas veces, $3=$ Algunas veces, $4=$ Muchas veces, $5=$ Siempre $)$. Puntuaciones más próximas a 5 indican mayores niveles en cada una de las variables.

$$
{ }^{\star \star} p<.01
$$

\section{Diferencias en autoeficacia en función del nivel de relaciones positivas con otros}

La Tabla 2 muestra las puntuaciones medias de los tres grupos (alto, medio y bajo) de relaciones positivas con otros en la variable autoeficacia general. Se observa que cuanto mayor es el grado de relaciones positivas con otras personas informado por los participantes del estudio, mayores son sus expectativas de autoeficacia. Los resultados del ANOVA muestran diferencias estadísticamente significativas en la variable autoeficacia entre los tres niveles de relaciones positivas con otros $(F(2,627)=30.21, p<.001)$. Atendiendo a los resultados de la prueba de contrastes a posteriori (Scheffé), se obtuvieron diferencias estadísticamente significativas de tamaño grande $(d=0.98)$ entre el grupo alto y el grupo bajo en relaciones positivas con otros. También resultaron estadísticamente significativas las diferencias entre el grupo alto y el grupo medio, siendo sus diferencias de tamaño medio ( $d=0.53$ ). Las diferencias entre el grupo medio y el grupo bajo también alcanzaron la significatividad estadística, si bien el tamaño del efecto fue pequeño $(d=0.44)$.

Tabla 2

Media y desviaciones típicas obtenidas por los distintos grupos de relaciones positivas con otros en autoeficacia

Autoeficacia

\begin{tabular}{ccccc}
\hline & Grupo & $N$ & $M$ & $D T$ \\
\hline & Bajo & 167 & 2.95 & 0.58 \\
Relaciones & Medio & 364 & 3.19 & 0.53 \\
positivas con & Alto & 99 & 3.49 & 0.54 \\
otros & Total & 630 & 3.18 & 0.57
\end{tabular}

Nota. Escala de medida de relaciones positivas con otros $(1=$ En total desacuerdo, $2=$ Algo en desacuerdo, $3=\mathrm{Ni}$ en desacuerdo ni de acuerdo, $4=$ Algo de acuerdo, $5=$ Totalmente de acuerdo); Escala de medida de autoeficacia $(1=$ Nunca, $2=$ Pocas veces, $3=$ Algunas veces, $4=$ Muchas veces, $5=$ Siempre $)$. Puntuaciones más próximas a 5 indican mayores niveles en cada una de las variables. 


\section{DISCUSIÓN}

Ryff $(1989,2013)$ ha evidenciado conceptual y empíricamente la importante contribución que hacen los vínculos significativos con otras personas para el bienestar psicológico. Adoptando un planteamiento concordante con esta perspectiva optimizadora del bienestar, en el presente estudio se pretendía determinar la existencia de diferencias significativas en función del grado (alto, medio y bajo) de relaciones positivas con otros respecto a la variable autoeficacia, considerada una de las variables más influyentes en la conducta motivada de los estudiantes (Alhadabi y Karpinski, 2020; Honicke y Broadbent, 2016; Schunk y Pajares, 2010).

De conformidad con la hipótesis de partida, los resultados de este trabajo sugieren una relación lineal positiva entre la variable relaciones positivas con otros y la variable autoeficacia en los estudiantes universitarios, de tal forma que, cuanto mayor es la primera, más elevada es la segunda, y viceversa. En concreto, nuestros hallazgos ponen de manifiesto la existencia de diferencias estadísticamente significativas en la autoeficacia percibida entre el grupo de estudiantes con altos niveles de vínculos sociales significativos y los estudiantes encuadrados en el grupo más bajo de esta variable. Estas diferencias, además, resultaron ser de una magnitud grande. También se obtuvieron diferencias significativas, aunque de tamaño mediano, entre los grupos alto y medio de relaciones positivas con otros. En conjunto, estos datos parecen ir en la línea de otros trabajos que asocian el apoyo social percibido por los universitarios con un mayor nivel de persistencia ante las dificultades (Mallinckrodt, 1988), un mejor afrontamiento de las demandas (DeRosier et al., 2013), así como una autoeficacia más elevada para tomar decisiones (Wright et al., 2014). El presente estudio permitiría profundizar en la comprensión de esta relación, en la medida en que los resultados obtenidos indican que el estudiantado universitario que posee sólidos lazos emocionales y desarrolla altos niveles de empatía y sentimientos de afecto por los demás muestra unos altos niveles generalizados de competencia autopercibida. Por el contrario, los niveles de autoeficacia general serían sensiblemente más bajos en los estudiantes con escasas y poco satisfactorias relaciones sociales y con dificultades para forjar vínculos sólidos con otras personas.

Habida cuenta de su relación con la autoeficacia, una importante implicación psicoeducativa que se deriva de estos hallazgos es la conveniencia de fomentar desde etapas educativas tempranas la capacidad de los estudiantes para establecer vínculos significativos con otras personas. Iniciativas como el Programa de Resiliencia de Pen (PRP) y el Plan de Estudios de Psicología de Strath Haven (Seligman, 2011) o el Programa Aulas Felices (véase García-Campayo, Demarzo y Modrego 2017) constituyen buenos ejemplos del trabajo educativo que se puede desarrollar en esta línea.

Sin desmerecer las aportaciones de este trabajo, es preciso tomar en consideración sus limitaciones a la hora de analizar los resultados obtenidos. Una primera limitación radica en el carácter transversal del diseño de investigación, el cual imposibilita el establecimiento de relaciones de causalidad entre las relaciones positivas con otras personas y la autoeficacia. Futuros trabajos deberán indagar en este posible vínculo causal mediante diseños de índole longitudinal. Como segunda limitación, debemos apuntar a las características de la muestra y al procedimiento de reclutamiento de los participantes, los cuales restringen la generalización de los hallazgos al conjunto de la población universitaria. En el futuro, otros estudios deberán corroborar los resultados obtenidos mediante procedimientos de muestreo más rigurosos. En tercer lugar, la utilización de procedimientos de autoinforme como método de recogida de datos puede suponer un sesgo para la obtención de datos veraces. Se precisan, por tanto, nuevos estudios que permitan confirmar estos hallazgos mediante la utilización de metodologías complementarias, tales como entrevistas en profundidad u observaciones in situ.

\section{REFERENCIAS BIBLIOGRÁFICAS}

Adams, G. R., Ryan, B. A., \& Keating, L. (2000). Family relationships, academic environments, and psychosocial development during the university experience: A longitudinal investigation. Journal of Adolescent Research, 15, 99-122. doi:10.1177/0743558400151006 
Alhadabi, A., \& Karpinski, A. C. (2020) Grit, self-efficacy, achievement orientation goals, and academic performance in University students. International Journal of Adolescence and Youth, 25(1), 519-535. doi:10.1080/02673843.2019.1679202

Baessler, J., \& Schwarcer, R. (1996). Evaluación de la autoeficacia: Adaptación española de la escala de Autoeficacia General. Ansiedad y Estrés, 2, 1-8.

Bandura, A. (1977). Self-efficacy: Toward a unifying theory of behavioral change. Psychological Review, 84(2), 191-215. doi:10.1037/0033-295X.84.2.191

Bandura, A. (1997). Self-Efficacy. The exercise of control. New Cork: W. H. Freeman and Company.

Baumeister, R. F., \& Leary, M. R. (1995). The need to belong: desire for interpersonal attachments as a fundamental human motivation. Psychological Bulletin, 117(3), 497-529. doi:10.1037/0033-2909.117.3.497

Cohen, J. (1988). Statistical power analysis for the behavioral sciences. Hillsdale, NJ: Erlbaum.

DeRosier, M., Frank, E., Schwartz, V., \& Leary, K. A. (2013). The potential role of resilience education for preventing mental health problems for college students. Psychiatric Annals, 43(12), 538-544. doi:10.3928/00485713-20131206-05

Díaz, D., Rodríguez-Carvajal, R., Blanco, A., Moreno-Jiménez, B., Gallardo, I., Valle, C., \& van Dierendock, D. (2006). Adaptación española de las escalas de bienestar psicológico de Ryff. Psicothema, 18(3), 572-577.

Feldman, L., Goncalves, L., Chacón-Puignau, G., Zaragoza, J., Bagés, N., \& de Pablo, J. (2008). Relaciones entre estrés académico, apoyo social, salud mental y rendimiento académico en estudiantes universitarios venezolanos. Universitas Psychologica, 7(3), 739-751.

Fiori, K. L., \& Consedine, N. S. (2013). Positive and negative social exchanges and mental health across the transition to college Loneliness as a mediator. Journal of Social and Personal Relationships, 30(7), 920-941. doi:10.1177/0265407512473863

Freire, C., \& Ferradás, M. M. (2019). Relación entre percepción de dominio del ambiente y autoeficacia en estudiantes universitarios. International Journal of Developmental and Educational Psychology INFAD Revista de Psicología, 1(4), 381-390. doi: 10.17060/ijodaep.2019.n1.v5.1613

Freire, C., Ferradás, M. M., Núñez, J. C., \& Valle, A. (2017). Estructura factorial de las Escalas de Bienestar Psicológico de Ryff en estudiantes universitarios. European Journal of Education and Psychology, 10(1), 18. doi:10.1016/j.ejeps.2016.10.001

García-Campayo, J., Demarzo, M., \& Modrego, M. (2017). Bienestar emocional y mindfulness en la educación. Madrid: Alizanza Editorial.

Honicke, T., \& Broadbent, J. (2016). The relation of academic self-efficacy to university student academic performance: A systematic review. Educational Research Review, 17, 63-84. doi:10.1016/j.edurev.2015.11.002

Lau, E. Y. H., Chan, K. K. S., \& Lam, C. B. (2018). Social support and adjustment outcomes of first-year university students in Hong Kong: self-esteem as a mediator. Journal of College Student Development 59(1), 129-134. doi:10.1353/csd.2018.0011

Mallinckrodt, B. (1988). Student retention, social support, and dropout intention: Comparison of black and white students. Journal of College Student Development, 29(1), 60-64.

Ritchie, L. (2016). Fostering self-efficacy in higher education students. London: Palgrave MacMillan.

Rowe, P. M. (1996). On the neurobiological basis of affiliation. APS Observer, 17-18.

Ryff, C. D. (1989). Happiness is everything, or is it? Explorations on the meaning of psychological well-being. Journal of Personality and Social Psychology, 57(6), 1069-1081. doi: 10.1037/0022-3514.57.6.1069

Ryff, C. D. (2013). Eudaimonic well-being and health: Mapping consequences of self-realization. En A. S. Waterman (Ed.), The best within us: Positive psychology perspectives on eudaimonia (pp. 77-98). Washington, DC: American Psychological Association.

Ryff C. D., \& Keyes, C. L. (1995). The structure of psychological well-being revisited. Journal of Personality and Social Psychology, 69(4), 719-727. doi: 10.1037/0022-3514.69.4.719 
Ryff, C. D., \& Singer, B. H. (2000). Interpersonal flourishing: a positive health agenda for the new millennium. Personality and Social Psychology Review, 4(1), 30-44. doi: 10.1207/S15327957PSPR0401_4

Sanjuán, P., Pérez, A. M., \& Bermúdez, J. (2000). Escala de autoeficacia general: datos psicométricos de la adaptación para población española. Psicothema, 12(2), 509-513.

Schnettler, B., Miranda-Zapata, E., Grunert, K. G., Lobos, G., Denegri, M., Hueche, C., \& Poblete, H. (2017) Life satisfaction of university students in relation to family and food in a developing country. Frontiers in Psychology, 8:1522. doi:10.3389/fpsyg.2017.01522

Schunk, D., \& Pajares, F. (2010). Self-efficacy beliefs. En P. Peterson, E. Baker y B. McGaw (Eds.), International Encyclopedia of Education, $3^{\text {rd }}$ ed. (pp. 668-672). New York: Elsevier.

Seligman, M. E. P. (2011). La vida que florece. Barcelona: Ediciones B.

Shih, H-J. (2019). L2 anxiety, self-regulatory strategies, self-efficacy, intended effort and academic achievement: A structural equation modeling approach. International Education Studies, 12(3), 24-35.

Wood, R., \& Bandura, A. (1989). Social cognitive theory of organizational management. Academy of Management Review, 14, 361-384.

World Medical Association (2013). World Medical Association Declaration of Helsinki: Ethical principles for medical research involving human subjects. JAMA, 310(20):2191-2194. doi:10.1001/jama.2013.281053

Wright, S. L., Perrone-McGovern, K. M., Boo, J. N., \& White, V. (2014). Influential factors in academic and career self-efficacy: Attachment, supports, and career barriers. Journal of Counseling \& Development, 92(1), 36-46. doi:10.1002/j.1556-6676.2014.00128.x

Zander, L., Brouwer, J., Jansen, E., Crayen, C., \& Hannover, B. (2018). Academic self-efficacy, growth mindsets, and university students' integration in academic and social support networks. Learning and Individual Differences, 62, 98-107. doi:10.1016/j.lindif.2018.01.012

Zumbrunn, S., Broda, M., Varier, S., \& Conklin, S. (2019). Examining the multidimensional role of self-efficacy for writing on student writing self-regulation and grades in elementary and high school. British Journal of Educational Psychology. doi: 10.1111/bjep.12315 